See discussions, stats, and author profiles for this publication at: https://www.researchgate.net/publication/224247139

\title{
Validation of a Model of a Resonant Optothermoacoustic Trace Gas Sensor
}

Conference Paper · June 2011

DOI: 10.1364/CLEO_AT.2011.JTUl115 · Source: IEEE Xplore

CITATION

READS

1

5 authors, including:

- J. Zweck

15 University of Texas at Dallas

76 PUBLICATIONS 966 CITATIONS

SEE PROFILE 


\title{
Validation of a Model of a Resonant Optothermoacoustic Trace Gas Sensor
}

\author{
N. Petra ${ }^{1}$, J. Zweck ${ }^{1}$, S. E. Minkoff ${ }^{1}$, A. A. Kosterev ${ }^{2}$, and J. H. Doty III ${ }^{2}$ \\ ${ }^{1}$ Department of Mathematics and Statistics University of Maryland Baltimore County, Baltimore, MD 21250, USA \\ ${ }^{2}$ Department of Electrical and Computer Engineering, Rice University, Houston, TX 77005, USA \\ E-mail: zweck@umbc.edu
}

\begin{abstract}
A model for a resonant optothermoacoustic sensor is validated by comparison with experiments performed with $0.5 \%$ acetylene in nitrogen. At low concentrations, the molecular dynamics of the trace gas do not influence the signal.

(C) 2010 Optical Society of America

OCIS codes: $300.6430,300.6340$.
\end{abstract}

\section{Introduction}

Quartz-Enhanced PhotoAcoustic Spectroscopy (QEPAS) and Resonant OptoThermoAcoustic DEtection (ROTADE) sensors are two recently developed methods that use a quartz tuning fork (QTF) to detect trace gases [1,2]. Both thermal diffusion and acoustic pressure waves can be generated when optical radiation is periodically absorbed by a trace gas. With QEPAS, the QTF responds to the pressure wave, whereas with ROTADE the diffusion wave is detected. QEPAS and ROTADE are complementary techniques. In fact, both processes occur simultaneously, although one is usually dominant [2]. At atmospheric pressure QEPAS dominates since the diffusion wave decays much more rapidly than does the acoustic wave. However, as the ambient pressure and density are lowered, the frequency of molecular collisions decreases, lowering the vibrational-to-translational relaxation rates and the efficiency of the conversion of molecular vibrational energy to acoustic energy. Therefore, ROTADE is more sensitive than QEPAS when the ambient pressure is low enough $(\lesssim 50$ Torr). At low ambient pressure the lines in the absorption spectrum become more distinct thus providing ROTADE sensors with more wavelength selectivity than other sensors.

In this paper, we introduce a computational model of a ROTADE sensor and use it to study how the amplitude and phase of the ROTADE signal depend on the position of the laser source. Our results show that the signal is largest when the laser is positioned near the base of the QTF. We validate the model by obtaining agreement with experimental results for $0.5 \%$ acetylene in nitrogen. Although a preliminary version of the model introduced in [3] was applied to compute the signal due to direct heating of a QTF by a laser, in this paper we present the first validation of a mathematical model of an actual ROTADE trace gas sensor.

ROTADE sensors are optothermal detectors. A laser generates optical radiation at a specific absorption wavelength of the trace gas to be detected. The laser beam is directed between the tines of a QTF (see Fig. 1 (left)). The absorbed optical energy is transformed into vibrational energy of the gas molecules and is transported to the surface of the QTF via molecular diffusion. Upon collision with the surface, the excited molecules transfer vibrational energy to the QTF in the form of heat. This heat then diffuses into the interior of the QTF where it induces a mechanical stress and displacement of the tines of the QTF. By modulating the wavelength (or power) of the laser, a mechanical resonance can be excited in the QTF. Since quartz is a piezoelectric material, this mechanical vibration can be converted into an electric current that is measured.

\section{Mathematical Model}

We use the heat equation to model both the diffusion of excited gas molecules to the surface of the QTF and the resulting diffusion of heat into the interior of the QTF. In particular, we ignore the dynamics of any energy exchange processes between the different degrees of freedom of the diffusing gas molecules. We also assume that all the energy of each gas molecule that is in excess of the ambient thermal energy is transferred to the surface in every collision, i.e., that the temperature is continuous across the surface of the QTF. Since the thermal source is time-harmonic, we solve for the amplitude, $T=T(\mathbf{x})$, of the thermal diffusion wave using the associated Helmholtz equation

$$
-\nabla \cdot(\kappa \nabla T)+i \omega T=S .
$$



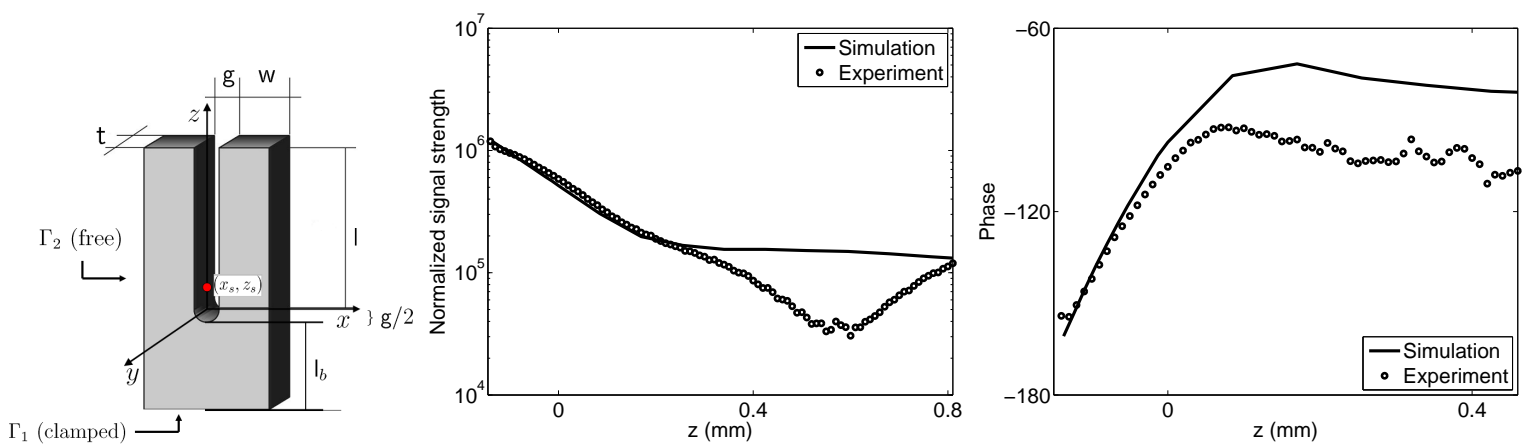

Fig. 1. Left: The tuning fork coordinate system. The laser beam axis is parallel to the $y$ axis and passes through the point $(x, z)=(0, z)$ on the vertical axis. The origin is on the axis of the semicircular surface (of radius $0.15 \mathrm{~mm}$ ) near the junction of the tines. The length, $l$, is $3.8 \mathrm{~mm}$. Center: Amplitude of the ROTADE signal as a function of laser source position, $z$. The solid lines correspond to numerical simulation results and the circles correspond to laboratory experiments. Right: Phase of the ROTADE signal.

Here $\kappa$ is the thermal diffusivity, $\omega$ is the modulation frequency of the laser (which equals the resonance frequency of the QTF), and $S$ is the amplitude of the thermal source, which is modeled using a Gaussian beam. The Helmholtz equation that models the temperature-driven elastic deformation of the QTF is given by

$$
\nabla \cdot C[\nabla \mathbf{u}]+\left(\rho \omega^{2}-i b \omega\right) \mathbf{u}=\nabla \cdot C[\alpha T] .
$$

Here $C$ is the elasticity tensor, $\rho$ is the density, $b$ is the damping coefficient, and $\alpha$ is the thermal expansion tensor of the QTF medium. The bottom surface of the tuning fork is fixed and the traction is set to zero on all remaining surfaces. We solve Eqns. (1) and (2) using the finite element method and calculate the piezoelectric signal from $\mathbf{u}$ as in [4].

\section{Results}

We validated the model by comparing results obtained from numerical simulations and laboratory experiments. These results were obtained for $0.5 \%$ acetylene in nitrogen at an ambient pressure of 20 Torr using the same QTF as in [4]. In Fig. 1 (center), we plot the amplitude of the ROTADE signal as a function of the vertical position, $z$, of the laser source (see Fig. 1 (left)). The amplitude curve from the simulations (solid line) was normalized to agree with the data from the experiments (circles) near $z=0$. Both results show that the ROTADE signal is largest when the laser source is positioned near the base of the QTF. In Fig. 1 (right), we plot the phase of the signal. The agreement near $z=0$ between the simulated and experimental signals (both in amplitude and in phase) provides a validation of the model. This result shows that the molecular dynamics of acetylene do not influence the signal. We expect a similar result to hold for many trace gases at low concentrations since in this situation collisions between excited molecules occur with negligible probability. We attribute the lack of agreement between the simulation results and the experimental data when $z>0.25 \mathrm{~mm}$ in Fig. 1 to the influence of the QEPAS signal, which we did not include in the model. In the experimental data, the behavior near $z=0.5 \mathrm{~mm}$ is due to destructive interference between the QEPAS and ROTADE signals [2]. In a future paper, the model will be used to numerically optimize the geometrical parameters of the QTF in a ROTADE sensor.

\section{References}

1. A. A. Kosterev, Y. A. Bakhirkin, R. F. Curl, And F. K. Tittel, Quartz-enhanced photoacoustic spectroscopy, Optics Letters, 27 (2002), pp. 1902-1904.

2. A. A. Kosterev AND J. H. Doty III, Resonant optothermoacoustic detection: A new technique for measuring weak optical absorption by gases and micro-objects, Optics Letters, 35 (2010), pp. 3571-3573.

3. N. Petra, A. A. Kosterev, J. Zweck, S. E. Minkoff, And J. H. Doty III, Numerical and experimental investigation for a resonant optothermoacoustic sensor, in Conference on Lasers and Electro-Optics, Optical Society of America, 2010, p. CMJ6.

4. N. Petra, J. Zweck, A. A. Kosterev, S. E. Minkoff, And D. ThomaZy, Theoretical analysis of a quartz-enhanced photoacoustic spectroscopy sensor, Appl. Phys. B, 94 (2009), pp. 673-680. 